Itinéraires Itinéraires

Littérature, textes, cultures

2020-2 | 2020

Discours animal. Langages, interactions, représentations

\title{
Imaginaires zoolinguistiques : des langues animales dans la fiction littéraire
}

Imaginary Zoolinguistics: Animal Languages in Fictional Literature

\section{Sophie Milcent-Lawson}

\section{(2) OpenEdition}

\section{Journals}

Édition électronique

URL : https://journals.openedition.org/itineraires/8352

DOI : $10.4000 /$ itineraires.8352

ISSN : 2427-920X

Éditeur

Pléiade

Référence électronique

Sophie Milcent-Lawson, «Imaginaires zoolinguistiques : des langues animales dans la fiction littéraire », Itinéraires [En ligne], 2020-2 | 2020, mis en ligne le 22 décembre 2020, consulté le 25 novembre 2021. URL : http://journals.openedition.org/itineraires/8352 ; DOI : https://doi.org/10.4000/ itineraires.8352

Ce document a été généré automatiquement le 25 novembre 2021.

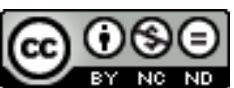

Itinéraires est mis à disposition selon les termes de la licence Creative Commons Attribution - Pas d'Utilisation Commerciale - Pas de Modification 4.0 International. 


\title{
Imaginaires zoolinguistiques : des langues animales dans la fiction littéraire
}

\author{
Imaginary Zoolinguistics: Animal Languages in Fictional Literature
}

\author{
Sophie Milcent-Lawson
}

«L'homme ne fait pas que parler les langues,

il les rêve aussi. » (Yaguello 2005 : 363)

1 Si les langues animales ont connu de multiples explorations fictionnelles, elles n'ont en revanche guère fait l'objet d'investigations linguistiques, et aucune enquête systématique ne leur a, à ce jour, été consacrée ${ }^{1}$. Ainsi de leur notable absence de l'étude de Marina Yaguello, Langues imaginaires, même dans sa version remaniée de 2006. Pourtant, par-delà les auteurs de science-fiction et de voyages imaginaires, des écrivains de toutes époques se sont essayés à imaginer de telles langues animales, depuis Aristophane (Les Grenouilles, Les Oiseaux, 405 et 414 av. J.-C.), les utopies des XVII et XVIII ${ }^{e}$ siècles (Cyrano de Bergerac, Restif de la Bretonne, Swift), en passant par les romans de Kipling, Lewis Carroll, la littérature du $\mathrm{xx}^{\mathrm{e}}$ siècle et jusqu'à la fiction ultracontemporaine. Loin de se contenter de « donner la parole » aux animaux en les faisant parler comme des humains, ces auteurs ont tenté de forger des uglossies, des langues animales se démarquant des langues humaines.

2 En marge d'une recherche sur les tentatives de représentation d'un point de vue animal en régime fictionnel ${ }^{2}$, cette étude se propose d'ouvrir la réflexion sur les imaginaires langagiers qui président aux représentations de langues animales imaginaires telles que la fiction littéraire a pu les concevoir au fil de diverses époques, aires linguistiques et champs esthétiques, de la satire à la fantaisie ludique en passant par la science-fiction. À partir de l'examen d'un échantillon de discours « animaux » tirés de la littérature, on s'efforcera donc de dégager les caractéristiques de cette zoolinguistique imaginaire.

3 Le choix dominant dans les fictions comportant des personnages animaux consiste à les faire s'exprimer en langage humain, souvent même sans que cette maîtrise d'une 
langue naturelle humaine ne soit questionnée par le récit (1. Les animaux "parlants » dans la littérature). Le second dispositif que l'on peut observer met en scène des locuteurs animaux malhabiles dans le maniement d'une langue humaine, ce qui donne lieu à une multiplicité d'effets, allant du comique disqualifiant à la fantaisie poétique jusqu'au questionnement philosophique sur ce que l'animalité fait à la langue (2. Dyscours animaux). Une troisième voie consiste enfin pour l'écrivain à se confronter au défi de l'invention d'une uglossie animale (3. Uglossies animales). L'examen de diverses tentatives permet de dégager quelques invariants linguistiques parmi ces langues animales fictives, esquissant les contours d'un imaginaire langagier (4. Imaginaires zoolinguistiques).

4 Faute de place, il ne s'agira pas de proposer un panorama exhaustif des diverses langues animales fictionnelles. L'ambition de ce rapide tour d'horizon sera plus modeste: donner quelques coups de sonde qui permettent d'ouvrir ce champ de recherches encore largement inexploré et proposer quelques pistes de réflexion.

\section{Les animaux « parlants » dans la littérature : l'animal locuteur d'une langue naturelle}

5 Nombre de fictions mettent en scène des animaux doués de parole sans qu'il soit nécessairement question d'univers merveilleux, de fantastique ni de science-fiction. La parole animale y est le plus souvent traitée comme allant de soi, elle n'est ni questionnée ni justifiée narrativement par un quelconque artifice de type anneau de Salomon ou métamorphose d'un humain enfermé dans un corps animal - justifiant par là même une pensée et un langage humain, comme c'est le cas dans L'Âne d'or d'Apulée, La Métamorphose de Kafka ou encore Truismes de Darrieussecq. Ainsi, chez La Fontaine, Colette (Dialogues de bêtes), Kafka (« Le Terrier »; « Les recherches d'un chien »), Orwell (La Ferme des animaux), Sewell (Black Beauty), Bach (Jonathan Livingstone le goéland) ou encore Hoffmann (Le Chat Murr) ou Sôseki (Je suis un chat) - pour ne citer que quelquesunes des œuvres les plus connues qui mettent en scène un animal parlant - les animaux locuteurs voire narrateurs font-ils montre d'une parfaite maîtrise d'une langue naturelle humaine. On trouvera parfois trace d'inflexions stylistiques figurant linguistiquement la nature animale de l'énonciateur, mais la langue utilisée n'en demeure pas moins une langue humaine. La narration recourt tout au plus à des marqueurs linguistiques destinés à rappeler au lecteur que locuteur est un animal. Ainsi Coeur de chien de Boulgakov s'ouvre-t-il sur une longue série onomatopéique imitant les pleurnichements du chien ("Ouh Ouh Ouh Ouh Ouh Ouh! Ouh! Ouh! Ouh!»), et si ses pensées sont ensuite retranscrites dans une langue non marquée du point de vue de l'espèce, son discours sera de temps à autre ponctué d'aboiements («Ouah! Ouah!» (p. 28) et de jappements (« Aouh! Aouh!, jappait-il tout bas », p. 35).

Douer les animaux de parole constitue un parti pris. L'aporie d'un discours animal qui n'emprunterait pas le matériel linguistique d'une langue humaine se trouve ainsi ignorée pour mieux la contourner, au prix d'une convention narrative qui relève du pacte de lecture implicite proposé à tout lecteur de fiction - au même titre par exemple que la convention du narrateur omniscient, instance tout aussi problématique du point de vue de la vraisemblance. Force est toutefois de constater que la convention de l'animal locuteur et la willing suspension of disbelief ${ }^{3}$ qu'elle exige eu égard à un cadre énonciatif non réaliste semble en butte à davantage de réticences - peut-être parce 
qu'élever l'animal au rang de locuteur c'est remettre en question la suprématie humaine et le privilège que s'accorde l'homme en tant qu'être de langage ${ }^{4}$. Faire parler l'animal comme un humain relève donc d'un vrai choix esthétique. En effet, malgré le réglage par nature humanocentré des langues naturelles, la langue littéraire constitue paradoxalement, par sa complexité même et la finesse de description et d'analyse qu'elle autorise, l'outil le mieux adapté à la saisie fine et précise des sensations, émotions et expériences mentales d'un animal - sa maîtrise linguistique de locuteur fictionnel fût-elle de pure convention.

\section{Dyscours 5 animaux : langue fautive}

7 Afin d'asseoir la vraisemblance interne à la fiction et de marquer que les langues humaines ne constituent pas leur mode naturel d'expression et de communication, les personnages d'animaux locuteurs ne font souvent valoir qu'une maîtrise partielle de la langue naturelle dans laquelle ils s'expriment. De nombreux auteurs de fiction font donc parler les animaux comme s'ils pratiquaient une langue étrangère qu'ils ne maitrisent pas tout à fait. Pour éviter que la maladresse linguistique qui en résulte ne compromette la lisibilité et la qualité littéraire de l'œuvre, ces locuteurs malhabiles ne se voient en général confier qu'une très faible portion du texte. En outre, les fautes de langue sont détournées tantôt au service du comique, tantôt reversées au compte d'une écriture poétique dont les anomalies se trouvent dès lors converties en inventivité linguistique.

\subsection{Paroles de chien par Rudyard Kipling (1930)}

Le recueil Paroles de chien (Kipling) rassemble sept brefs textes initialement parus en revue en $1930^{6}$. La langue supposée parlée (ou pensée) par les chiens se caractérise par son approximatisme grammatical et lexical. Elle s'apparente à un parler enfantin. Les mots subissent aussi des déformations phonétiques (par exemple «dretful» mis pour dreadful, traduit par "horribe» en français). Le procédé le plus récurrent soude en suites polylexicales figées des périphrases destinées à combler les lacunes lexicales tout en rendant compte du point de vue du chien : ainsi les veaux sont-ils des « chiots-devache » (cow pups), la voiture une «niche-qui-bouge » (kennel-that-moves) ou le berceau du bébé une "très petite niche-qui-se-balance " (kennel-that-rocks). Ces enfantillages canins ont certes quelque chose de charmant où transparaît toute l'affection que l'auteur portait à ses chiens, mais on ne peut s'empêcher d'y sentir également une forme de condescendance anthropocentrique: derrière la naïveté infantile de cette langue prêtée aux personnages de Boots, Slippers et ses amis, s'affirme aussi leur incapacité à saisir la complexité du monde qui les entoure. Le regard tendre et complice porté sur les jeunes chiens bagarreurs et désobéissants peine à faire oublier ce préjugé que les balourdises linguistiques emblématisent.

Les altérations syntaxiques et lacunes de vocabulaire prêtées aux locuteurs animaux peuvent toutefois servir de tout autres enjeux comme donner une forme fictionnelle à une réflexion sur les questions zoolinguistiques. 


\subsection{Le monkage de Doogie dans Mémoires de la jungle}

motive le projet romanesque de Tristan Garcia dans Mémoires de la jungle, paru chez Gallimard en 2010. Doogie a été éduqué dans une famille de scientifiques qui lui a enseigné la langue des signes américaine. Or, ses aptitudes exceptionnelles sont telles qu'il est à même d'assurer le rôle de narrateur de sa propre histoire. L'imaginaire de la langue que déploie fictionnellement Tristan Garcia s'efforce de penser l'influence que le statut de locuteur non-humain exerce sur la physionomie de la langue telle qu'il la parle - en altérant la syntaxe, le lexique, la prosodie, la grammaire - et, réciproquement, la violence que constitue pour un animal de devoir se couler dans les structures de pensée anthropologiques véhiculées par le langage humain. Une telle réflexion est tout à fait inédite dans le corpus fictionnel des animaux parlants et relaie les interrogations cognitives et éthiques de l'époque contemporaine qui voit vaciller la frontière entre animaux humains et non humains.

11 À l'image de son histoire, qui a fait d'un jeune animal sauvage un être hybride bloqué sur le seuil entre animalité et humanité, la langue de Doogie est hybridée par sa nature simienne. La langue narrative des Mémoires de la jungle connaît donc une syntaxe particulièrement malmenée. La subversion des catégories morphosyntaxiques et de l'ordre des mots confère au discours du chimpanzé une prosodie singulière: «À randonne, je brouillard, je brume, je clair, ça travers taillis tes parasites » (342). Si la matérialité sonore de ce monkage est clairement survalorisée, avec engendrement du discours par attraction phonique et rythmique («Quand nuit, je niche, je nid », p. 342), ces paronomases se doublent de motivations sémantiques (« Ni dit ni rêve, à l'allonge, sans nid cent nuits, tout vit sauf moi. Sauve-moi», p. 344) voire d'accents métaphysiques : " Ici rien n'esprit, rien n'est de tout fut, mes morts, mes moues, des ans, néant " (336). Les constructions fautives peuvent donc faire l'objet d'une double lecture dans la mesure où certaines infractions grammaticales se teintent de connotations poétiques : « le ciel pleure » (104), « bientôt la nuit a froid » (114). Il en va de même des lacunes lexicales, que comblent des périphrases métaphoriques qui manifestent à la fois sens de l'observation et sens poétique. Ignorant le mot interrupteur, Doogie formule par exemple : "je cherche le oui ou le non de la lumière » (119). Il est également difficile de démêler si les traits d'humour portés par son discours chaotique sont ou non volontaires, mais on peine à croire qu'il n'y ait aucune malice dans les désignations fautives que Doogie s'obstine à appliquer aux bonobos et macaques qu'il appelle bonobob (237) et macaca (129).

La langue imaginaire du narrateur chimpanzé Doogie fonctionne donc simultanément comme un exercice de style et comme une réflexion en actes sur l'appropriation par l'animal d'un mode d'expression qui n'est pas le sien et qu'il doit réinventer à son usage s'il veut se constituer comme sujet - et non pas rester enfermé dans la langue du « maître ». Dans cette fiction, le langage humain apparaît dès lors pour la première fois comme un instrument de possible oppression - et non comme un outil libérant une parole animale qui serait, sans elle, privée de langage. 


\title{
3. Uglossies animales : du phonomimétisme au phonologique
}

\author{
«Combien faut-il de mots ou de sons distincts \\ pour mériter le nom de langage?» \\ (Vercors, Les Animaux dénaturés, 1952, p. 58)
} uglossies animales? Dans un premier type d'invention, le lexique s'efforce vers un phonomimétisme censé évoquer le cri ou le chant de l'animal. Mais dans quelle mesure cette «onomatopoétique» (Genette 1976: 149-182) mimétique des vocalisations animales est-elle apte à engendrer un véritable système phonologique?

\subsection{Aristophane : Les Oiseaux et Les Grenouilles}

Dans la comédie d'Aristophane Les Oiseaux (414 av. J.-C.), le personnage de La Huppe jadis homme et désormais oiseau - s'exprime en langue grecque non fautive mais il parsème ses propos de séquences onomatopéiques :

PISTHETAIROS - La huppe s'apprête à son tour à chanter.

LA HUPPE - Epopoi, popoi, popopopoi popoi, io, io. Ici, ici, ici, chacune de mes compagnes ailées! [...] tio, tio, tio, tio, tio, tio, tio, tio, et vous qui vivez dans les jardins, sur les rameaux de lierre, et vous hôtes des montagnes, vous, friands de l'olive sauvage, vous mangeurs d'arbouses, hâtez-vous de voler vers mon chant: trioto, trioto, totobrix [...] Allons, venez en masse exprimer vos avis, ici, ici, ici, ici. Torororo rorototorotix, kikkabau, kikkabau, torotorotorotorolililix. (36-37)

Dans Les Grenouilles (405 av. J.-C.), les batraciens se vantent de l'harmonie de leur chant :

LES GRENOUILLES - Brékékékex, coax coax, brékékékex, coax coax, filles des sources marécageuses, faisons entendre à l'unisson nos hymnes, mon chant harmonieux, coax, coax, que nous faisons retentir au Marais en l'honneur de Dionysos de Nysa, lorsque, à la fête des marmites, la foule des festoyeurs en état d'ivresse pénètre en mon domaine sacré. Brékékékex, coax coax. (244)

Dionysos se moque d'elles ( Puissiez-vous crever avec votre coax, car vous n'êtes que coax!»(244)) et reprend en écho "Brékékékex, coax coax», assimilant ce que les grenouilles présentent comme un chant harmonieux à des pets, dont il accompagne sa propre singerie. Rien ne permet donc d'affirmer que les suites onomatopéiques représentent autre chose que des émissions vocales, et non des signes linguistiques pourvus de sens. C'est ce que laissait aussi entendre la réplique qui précède celle de la Huppe et qui annonçait un chant, ou ailleurs la mention par le chœur d'un chant d'allégresse en commentaire des ponctuations phonétiques en tiotiotiotrix (p. 59). Le pas entre phonosphère et sémiosphère ne semble pas franchi.

\subsection{Restif de la Bretonne (1781)}

Dans sa fiction utopique La Découverte australe par un Homme-volant, Restif de la Bretonne imagine la visite successive d'une série d'îles peuplées par des êtres mihumains mi-animaux. Parmi eux, les hommes-lions, dont le narrateur s'attarde à décrire la langue :

Or il faut vous dire que durant le traitement, Hermantin et ses compagnons étaient parvenus à enseigner un peu de français aux blessés, et à comprendre quelquesunes de leurs expressions. Elles étaient fort grossières, et leur langue ne consistait 
guère qu'en vingt ou trente mots, sans particules ni liaison. M'r'hô-on-hhom signifiait du gibier; r'hhhômb, courir ; hhoûhhamp, attraper ; hhîhhoûmhp, aimer, désirer, vouloir; fhlloûfhlloûp, du sang. Les noms n'y ont que le vocatif, et les verbes l'infinitif seul: un geste en devant marque le futur, un geste en arrière exprime le passé, la griffe sur la tête est pour le présent: il n'y a que ces temps-là. Point d'adjectif, que bon et mauvais, long et court, encore ne sont-ce des mots mais des signes : le premier consiste à poser la main à plat sur le cœur ; le second à l'éloigner de soi ; le troisième se trace sur la main depuis le poignet jusqu'au bout des doigts ; pour le quatrième on met un doigt sur la pointe de la griffe; ce signe signifie aussi bientôt, sur l'heure, aujourd'hui, etc., comme long marque le contraire. Le sentiment de haïr, d'abhorrer n'est qu'un geste, avec un cri, mm'hoûmp! qui sans geste, veut dire refuser, ne vouloir pas, fuir, etc. Cette langue est donc aussi facile à apprendre, qu'imparfaite et peu expressive; mais les gestes ne laissent pas que de la rendre capable d'exprimer assez de choses pour que des êtres intelligents puissent rendre par elle toutes les idées de la vie commune. (Restif de la Bretonne [1781] 1990 : 1215)

L'échantillon suffit à exemplifier la composante onomatopéique et la grammaire élémentaire de la langue de ces Hommes-Bêtes. Si la tentative demeure une ébauche rapide, elle illustre bien un imaginaire zoolinguistique préjugeant d'une part d'un degré d'élaboration moindre de ces langues animales comparées aux langues humaines, et d'autre part reposant sur une structuration phono-morphologique qui s'interdit toute ressemblance avec des idiomes existants.

\subsection{Watership Down (1972)}

Dans le roman pour la jeunesse de Richard Adams qui raconte les aventures épiques des lapins de la garenne de Watership Down, le récit est très largement rédigé en anglais standard. Cependant, la présence d'un petit nombre de termes en lapine, la langue fictionnelle des lapins imaginée par l'auteur, a pour fonction de figurer linguistiquement l'appartenance des personnages de la fiction à une autre espèce. Si les noms des principaux protagonistes adoptent une morphologie composite qui emprunte à de nombreuses langues (Hazel, Fyveer, Pikyn, Akraan, Dandelion, Bigwig, Keehar, Rahmnus, Primerol, Fraga et sa hase Nildo-hann, etc.) Adams s'est efforcé de donner une réalité lexicale à cette langue lapine. Il la dote aussi de quelques traits grammaticaux comme un morphème de pluriel : ainsi yona (« le hérisson ») s'oppose à yonou (197). Les termes figurant en langue lapine dans le texte incarnent, à la manière de xénismes, des réalités propres au monde de la garenne, à l'image du mot sfar qui désigne le fait d'être paralysé par la peur - sentiment emblématique du peuple lapin, du verbe farfal[er] (102) signifiant "remonter à la surface, farf, pour se nourrir, fal » (102) -, ou encore de notions comme vilou (passim), qui désigne toute forme d'ennemi, ou encore skramouk (dont une note précise que «ce mot désigne l'odeur du renard et s'applique à tout ce qui inspire la répulsion ", p.49). Le lapine répond donc au modèle d'une languenomenclature, où à chaque mot correspond une idée. L'auteur explicite le plus souvent les nouveaux mots en note de bas de page, à la façon d'un traducteur. Le lapine exprime enfin une vision du monde décentrée, non-anthropocentrique. Adams imagine par exemple une façon de compter propre aux lapins :

Les lapins ne savent compter que jusqu'à quatre, au-delà tout est shraar, que ce soit beaucoup ou mille. Tchoun au contraire veut dire petit. Donc le nom de Shraar-

Tchoun, qui est sans doute né d'une portée de plus de quatre lapereaux, peut se comprendre comme: "Petit Mille», pas grand-chose d'un ensemble plus grand, l'avorton. (12)

Ce faisant, le romancier animalier se fait à la fois philologue et ethno-zoologue. 


\subsection{Un idiome : la langue des Houyhnhnms de Swift} qui nourrissait un goût avéré pour la création linguistique et était féru des inventions rabelaisiennes (Pons 1976 : 394) élabore un idiome pour son peuple cheval. À l'écrit, cette langue se signale principalement par l'insertion des lettres $h, h n, l h, y$ et $w$ qui déguisent graphiquement les matrices homo/homme en Houyhnhnms ou en hhnm, ou le pronom de première personne yo - qui stigmatise l'égocentrisme des humains - en Yahoo. Si la présence massive des groupes $h$ et $h n$ participent à l'évidence d'un phonosymbolisme évocateur du hennissement, ces altérations par saupoudrage de lettres adventices répondent tout autant à une logique de cryptage, ces jeux graphiques parodiant une orthographe irlandaise hybridée de langue celtique. Ces déformations phonétiques et graphiques n'obéissent donc pas d'abord au souci d'imaginer une langue animale. Conformément aux autres épisodes du conte philosophique, l'invention de ce peuple fictif de chevaux policés vise l'ethnocentrisme européen et procède, via le regard exempt de préjugés porté par ces peuplades lointaines, à une violente satire des sociétés occidentales. L'absence de certains mots dans la langue des chevaux trahit donc leur supériorité morale : «Il répétait que je me trompais sûrement ou que je disais la-chose-qui-n'est-pas (car ils n'ont pas de mots dans leur langue pour désigner le mensonge ou les faussetés) »(292). La liste est longue des notions qui n'ont ainsi aucune existence pour eux : "Pouvoir, administration, guerre, loi, châtiment et mille autres termes n'avaient aucun équivalent dans leur langue » (303). Dès lors seul le nom de ces humains dépravés qu'ils appellent Yahoos, peut faire office de suffixe dépréciatif :

Il est peut-être inutile de faire remarquer que les Houyhnhnms n'ont pas dans leur langue de mots pour exprimer l'idée de mal, sauf ceux que leur suggèrent les laideurs physiques et morales des Yahoos. Ainsi quand ils veulent parler de la sottise d'un laquais, de l'étourderie d'un enfant, d'une pierre aiguë qui les a blessés au pied, d'une longue période de temps désagréable ou hors de saison, ils disent tous ces mots suivis de l'épithète yahoo. Par exemple : Hhnm yahoo, Whnaholm yahoo, Ynlhmndwihlma yahoo et une maison inconfortable : Ynholmhnmrolnw yahoo. (343)

La principale originalité de cette langue imaginaire animale au sein de notre corpus réside dans le fait que Gulliver, le narrateur humain, non seulement est désireux de l'apprendre pour communiquer avec ceux qu'il appelle ses "maîtres", mais encore qu'il lui accorde une supériorité sur, dit-il, «notre barbare langue anglaise » (304). Le renversement du préjugé anthropocentrique n'est dès lors qu'une métaphore de la remise en question de l'illusion ethnocentrique, car la réflexion de Swift ne porte pas réellement sur les rapports homme/animal et ses Houyhnhnms sont des chevaux on ne peut plus anthropomorphiques.

\subsection{Le Doggee de Lewis Carroll}

19 Sylvie et Bruno, troisième et dernier roman de Lewis Carroll, comporte un épisode intitulé « A visit to dogland» («Une visite en territoire canin»; Carroll $2003: 492-499$ ). À l'entrée du royaume, les enfants sont accueillis par un gros mastiff, chien d'une race anglaise voisine des dogues :

"Oobooh, hooh boohooyah"! He growled at last. "Woobah yahwah oobooh! Bow

wahbah woobooyah? Bow wow?" He asked Bruno, Severely. (Carroll 1988)

Itinéraires, 2020-2 | 2020 
« ouh, ouah, bou, ouah! » grogna-t-il enfin. « Ouw, ouh ouah yah oubou? Bou woua

wouhboua? Bou oua? » demanda-t-il sévèrement à Bruno. (Carroll $2003: 492$ )

Le texte comporte donc quelques fragments en langage chien, le doggee, que les jeunes héros comprennent sans difficulté : "Bien sûr Bruno comprenait tout cela assez facilement. Toutes les fées connaissent le langage canin. Mais comme vous pouvez le trouver un peu difficile au début, je préfère vous le mettre en français ", nous concède aimablement le narrateur qui traduit pour le lecteur les propos de la sentinelle : «Des humains, je crois bien! Une paire d'humains égarés! À quel chien appartenez-vous? Que voulez-vous?»(Carroll 2003 : 492-493).

Or, la comparaison entre les textes anglais et français fait clairement apparaître que la traduction française ${ }^{7}$ n'a pas considéré le doggee comme une langue mais comme une simple séquence d'onomatopées mimant approximativement des aboiements. C'est la raison pour laquelle, les onomatopées conventionnelles n'étant pas les mêmes en anglais et en français, le texte en doggee se trouve sensiblement modifié dans la version en français. C'est n'avoir pas perçu que bow wow, même s'il est bien une onomatopée conventionnelle en anglais ${ }^{8}$, s'était ici vu assigner une fonction sémantique puisqu'elle formule une question qui compte deux occurrences dans le passage, et dont seule la première fait l'objet d'une traduction : "What do you want?» (Que voulez-vous ?). Le terme bow apparaissait déjà en tête de la question précédente "Bow wahbah woobooyah?", ce qui laisse entendre qu'il est un équivalent canin du pronom interrogatif. Lewis Carroll a converti la transcription onomatopéique d'un cri en signifiant stable doté d'un sémantisme. Or, le texte français transpose Bow une première fois en Bou puis Bou oua?, tandis que la seconde occurrence de la même question est bizarrement maintenue dans le texte français sous sa forme anglaise Bow wow, interdisant au lecteur français de comprendre la seconde question posée par le Roi du territoire canin, puisqu'il n'est plus en mesure de l'identifier à celle déjà posée par le dogue à l'entrée du palais. Le même phénomène se répète au sujet de plusieurs termes, considérés à l'évidence comme de simples onomatopées par la traductrice française qui les répartit un peu au hasard là où le texte anglais permettait de repérer des récurrences qui les signalent comme des morphèmes à part entière. Ainsi par exemple de Oohbooh, terme canin désignant les humains.

21 Ces observations ne seraient qu'anecdotiques si elles ne trahissaient que la traductrice n'a pas accordé le statut de langue au doggee, alors même que Lewis Carroll a pris grand soin de construire un lexique et une grammaire pour les quelques bribes de sa langue imaginaire. C'est manifestement la proximité formelle des signifiants avec les onomatopées conventionnelles qui a empêché la traductrice de repérer sous leur distribution savante leur conversion en morphèmes ${ }^{9}$. Queneau l'a bien remarqué, lui qui se livre à propos de ce texte à un savoureux pastiche d'étude linguistique du langage chien. 


\title{
4. Imaginaires zoolinguistiques : glossologie ou glossolalie?
}

\subsection{Raymond Queneau : «De quelques langages animaux imaginaires et notamment du langage chien dans Sylvie et Bruno » (1971)}

\author{
«Quels sont les rapports de ce langage chien \\ imaginaire avec le langage chien réel ? Voilà la \\ question » \\ (Queneau [1971] 1995 : 104)
}

L'essai de Queneau obéit à des motivations parodiques évidentes. Il s'y livre malgré tout à une analyse attentive des caractéristiques linguistiques du doggee :

Lewis Carroll procure un corpus de neuf phrases qui permet d'identifier dix-huit mots différents dont on peut découvrir le sens grâce à la traduction qu'il en donne. [...] Au point de vue phonétique, le langage chien comporte deux consonnes (B et $\mathrm{H})$, deux semi-voyelles ( $\mathrm{Y}$ et $\mathrm{W}$ ) et deux voyelles (A et $\mathrm{O}$ ). [...] On notera aussi, toujours au point de vue phonologique, que $66,66 \%$ des mots se terminent par ooh, et $11 \%$ par ow. Par contre le W est le plus souvent à l'initiale $(38,88 \%)$ suivi par l'H (27,77 \%) et l'o (5,55\%). (102-103)

Queneau poursuit par une analyse grammaticale et syntaxique :

La grammaire semble assez proche du chinois ; pour autant qu'on puisse l'affirmer avec un matériel aussi restreint, il n'y a ni déclinaison ni conjugaison. Quant à la syntaxe, elle présente certaines particularités typiques de l'anglais, par exemple la tournure interrogative "Sujet+être+négation+adjectif, être+pronom » se retrouve en langage chien. (103)

Toutes ces observations minutieuses sont exactes ${ }^{10}$. Queneau remarque que la syntaxe du doggee est calquée sur celle de l'anglais, avec présence du question-tag en fin de phrase interrogative (hah bah, "is she?»). Il aurait tout aussi bien pu relever l'antéposition de l'adjectif (oohboh en fin de phrase traduisant «humain", il y a fort à parier que la forme qui se trouve placée à sa gauche traduit l'adjectif composé anglais good-looking donné dans la traduction, et que donc le mot hoobah est construit sur le même modèle).

Là où il fait preuve d'une intuition à la fois moqueuse et prophétique, c'est lorsqu'il propose de confronter la validité de cette « langue chien » imaginaire avec « le langage chien réel ", citant à l'appui de sa démonstration une étude d'un certain François Rostand (Développement de l'aboi chez un jeune chien, méthodes d'étude et premiers résultats), dont il ne nous a pas été possible de déterminer s'il s'agit d'une étude réelle ou d'une invention ${ }^{11}$. Si Queneau commente les divergences de pourcentages entre «monosyllabes, dissyllabes, trisyllabes et tétrasyllabes ou plus» dans les corpus produits par Carroll et Rostand, c'est pour en tirer une conclusion toute parodique : «Les divergences que l'on peut remarquer peuvent s'expliquer soit par le fait que les sujets de Lewis Carroll sont des adultes" (alors que l'étude prétendument tirée du Journal de psychologie normale et pathologique porte sur le «jeune chien»), «soit par la britannicité des uns et la velchité des autres»(105), postulant ainsi des variations dialectales. C'est anticiper, sur le mode du non sérieux, les études récentes en bioacoustique. Or, un siècle avant Queneau, un polygraphe belge touche-à-tout appelait lui aussi de ses vœux une documentation scientifique sur les vocalisations animales. 


\subsection{Pierquin de Gembloux : Idiomologie des animaux et glossaire ouistiti (1844)}

24 Le texte parodique de Queneau a tous les traits d'un discours savant, et c'est là ce qui fait tout son sel. L'essai de Claude-Charles Pierquin de Gembloux, Idiomologie des animaux ou Recherches historiques, anatomiques, physiologiques, philologiques, et glossologiques sur le langage des bêtes se présente quant à lui comme un essai tout à fait sérieux. En fin d'ouvrage, figure pourtant un "glossaire ouistiti » dont les entrées alphabétiques font l'objet de commentaires mobilisant les compétences linguistiques de l'auteur :

GUENOKIKI. - Frayeur terrible: cri d'alarme qui équivaut à fuir, à craindre fortement, redouter. - La prononciation de la première syllabe est fortement gutturo-nasale.

IROUAHHI. - Douleur violente et morale allant jusqu'au désespoir. - Prononciation gutturo-nasale.

IROUAH-GNO. - J'ai une douleur morale affreuse, sauvez-moi, épargnez-la moi. Prononciation gutturo-nasale très-prononcée.

KRRRREOEOEO. - Être heureux, jouir d'un bonheur profond, accompli; prononciation surlaryngienne aiguë quoique faible, tremblottante, et grasseyant. Cette exclamation, ou ce substantif verbal, est également répété plusieurs fois de suite et d'autant plus fortement que la joie qui la fait pousser est plus vive et plus grande.

KEH. - Être un peu mieux, souffrir moins. - Prononciation gutturale. (Pierquin de Gembloux [1844] 2016 : 1631-1678)

Son étude se termine sur ce vœu :

Il serait à souhaiter qu'un certain nombre d'hommes éclairés s'adonnât à l'étude philologique d'une tribu zoologique, que d'autres contrôleraient, et de la réunion des observations diverses résulteraient des vocabulaires complets. (Pierquin de Gembloux [1844] 2016 : 1678-1680)

La parodie de Queneau, l'imagination de Lewis Carroll et le sérieux fantaisiste de Gembloux convergent ainsi dans une même curiosité conçue pour les langues animales, curiosité annonciatrice des recherches actuelles menées par les bio-acousticiens et ethnozoologues dans le champ en pleine expansion de la zoosémiotique ${ }^{12}$.

\section{Langues animales imaginaires et imaginaires zoolinguistiques : un bilan}

L'extrême dispersion des corpus des langues animales masque leur présence tout au long de l'histoire de la littérature, de sorte qu'elles n'ont guère jusqu'à présent retenu l'attention des chercheurs ${ }^{13}$. Leurs actualisations témoignent en outre de la difficulté qu'il y a à forger de toutes pièces une langue imaginaire, difficulté encore accrue quand se manifeste l'ambition de sortir de l'anthropocentrisme linguistique manifesté par le logocentrisme et même le phonocentrisme, qui réduisent la notion de langage à celle de langage verbal quand d'autres systèmes sémiotiques (visuels, tactiles mais aussi olfactifs) sont aptes à véhiculer du sens et à servir de base à un système de communication interindividuelle. Les langues animales fictionnelles demeurent donc très largement un paradigme absent, latent, ou au mieux lacunaire. Les tentatives littéraires se bornent le plus souvent, en l'absence d'une grammaire, à l'invention d'un lexique empruntant son matériel phonologique aux langues humaines, tout en 
cherchant à s'en distinguer par des combinaisons de phonèmes inattendues mais dictées dans une large mesure par une survalorisation des préoccupations mimétiques. Les fictions linguistiques qui se risquent à un retranchement délibéré hors de la parole articulée sont plus rares encore. Les audaces d'un Cyrano de Bergerac (L'Autre monde, 1655), s'essayant à une sortie du phonocentrisme lorsqu'il imagine un langage musical ou dansé n'ont guère connu de continuateurs; par ailleurs la substitution de l'articulation par des gesticulations n'est pas sans comporter une dimension satirique ${ }^{14}$. Cette fiction du XVII siècle demeure toutefois l'une des rares à inverser l'orientation satirique pour dénoncer l'arrogance des humains qui s'arrogent une prééminence sur la foi de leur maîtrise de la parole alors même qu'ils s'avèrent incapables de comprendre les langages des autres vivants, qui eux, semblent capables de comprendre les hommes.

Il convient pour finir de remarquer que les langues imaginaires animales trouvent essentiellement place dans les fictions de voyage en pays lointains et finalement peu dans l'exploration des modes de communication des animaux qui partagent notre espace de vie. En science-fiction, les projections dystopiques trahissent l'angoisse de voir l'humanité supplantée par une autre espèce plus qu'elles ne témoignent d'un réel intérêt pour les mondes animaux qui coexistent avec nous sur la planète. Bref, la représentation de langues animales imaginaires ne répond pas essentiellement à ce qui serait une authentique curiosité pour les langages animaux et à travers eux, pour leurs univers mentaux. Mais il $\mathrm{y}$ a fort à parier que les avancées récentes en éthologie contribueront à modifier et à enrichir ces imaginaires zoolinguistiques.

\section{BIBLIOGRAPHIE}

Albani, Paolo et Buonarroti, Berlinghiero, [1994] 2001, Dictionnaire des langues imaginaires, trad. fr. par Egidio Festa, Paris, Les Belles Lettres.

Christen, Yves, 2013, L'Animal est-il un philosophe ? Poussins kantiens et bonobos aristotéliciens, Paris, Odile Jacob.

Genette, Gérard, 1976, Mimologiques. Voyage en Cratylie, Paris, Seuil, coll. « Poétique ».

Jeandillou, Jean-François, 1985, « Queneau ou le dyscours de la linguistique », dans S. Auroux, J.C. Chevalier, W. Jacques-Chaquin et C. Marchello-Nizia (dir.), La linguistique fantastique, Paris, Denoël, p. 211-221.

Landragin, Frédéric, 2018, Comment parler à un alien ? Langage et linguistique dans la science-fiction, Saint-Mammès, Éditions du Bélial, coll. « Parallaxe ».

Matignon, Karine-Lou, 2018, Paroles d'animaux. Écouter ce qu'ils ont à nous dire, Paris, La Découverte, Arte éditions.

Milcent-Lawson, Sophie, 2017, « Zoographies. Traitements linguistique et stylistique du point de vue animal en régime fictionnel », Revue des Sciences Humaines, $\mathrm{n}^{\circ} 328$, « Zoopoétique : les 
animaux dans la littérature de langue française ( $\mathrm{xx}^{\mathrm{e}}-\mathrm{xxI}^{\mathrm{e}}$ siècles) ", Anne Simon et André Benhaïm (dir.), p. 91-106.

Parmentier, Bérengère, 2004, « Parler sans la langue. Langages et corps dans Les États et Empires de Cyrano de Bergerac », Littératures Classiques, n 52, p. 219-236.

Pons, Émile, 1976, « Notes sur les procédés swiftiens de création linguistique dans les Voyages de Gulliver », dans Voyages de Gulliver, Paris, Gallimard, coll. « Folio », p. 394-397.

Simon, Anne, 2017, «La zoopoétique, une approche émergente : le cas du roman », RSH, $n^{\circ} 328$, p. 71-89.

Yaguello, Marina, 2005, «L'invention des langues », dans J.-M. Hombert (dir.), Aux origines des langues et du langage, Paris, Fayard, p. 362-389.

Yaguello, Marina, [1984] 2006, Les Langues imaginaires. Mythes, utopies, fantasmes, chimères et fictions linguistiques, Paris, Seuil.

\section{Corpus}

Adams, Richard, Watership down, [1972 ; trad fr. 1976] 2016, s. 1., éditions Monsieur Toussaint Louverture.

Aristophane, Les Oiseaux, 1966, dans Théâtre complet 2, traduction par Marc-Jean Alfonsi, Paris, Garnier Frères, coll. « GF », p. 23-98.

Aristophane, Les Grenouilles, 1966, dans Théâtre complet 2, traduction par Marc-Jean Alfonsi, Paris, Garnier Frères, coll. « GF », p. 231-297.

Boulgakov, Mikhail, [1925] 2012, Cœur de chien, Paris, Éditions Sillage, traduit du russe par M. Roman.

Carroll, Lewis, [1990] 2003, Sylvie et Bruno, chapitre XIII « Une visite en territoire canin », Euvres Complètes, t. II, Paris, Gallimard, coll. «Bibliothèque de la Pléiade », p. 492-499.

Carroll, Lewis, [1889] 1988, Sylvie and Bruno, [Londres, Mc Millan and co.], Dover publications, N.Y., USA.

Carroll, Lewis, 1982, La Revanche de Bruno, traduit de l'anglais par Pierre Leyris, Bordas.

Cyrano de Bergerac, Savinien, [1655] 2004, L'Autre monde. Les États et Empires de la lune. Les États et Empires du soleil, éd. de Jacques Prévot, Paris, Gallimard, coll. « Folio classique ».

Garcia, Tristan, 2010, Mémoires de la jungle, Paris, Gallimard, coll. « Folio ».

Kipling, Rudyard, [1894] 2000, « The white Seal », The Jungle Book, in The Jungle Books, Londres, Penguin Books, « Penguin Classics ».

Kipling, Rudyard, [1899] 1994, Le Livre de la jungle, Paris, GF-Flammarion, trad. fr. Louis Fabulet et Robert d'Humières [Mercure de France, 1899], édition Alexis Tadié.

Kipling, Rudyard, 2016, Thy Servant a Dog and other Dog Stories, [1930], The University of Adelaide Library, format Kindle.

Kipling, Rudyard, 2010, Paroles de chien, traduction française par T. Gillyboeuf, Paris, Rivages Poche, Petite bibliothèque.

Pierquin de Gembloux, Claude-Charles, [1844] 2016, Idiomologie des animaux ou Recherches historiques, anatomiques, physiologiques, philologiques, et glossologiques sur le langage des bêtes [Paris, 
La Tour de Babel, 1844], édition numérique collection XIX-Bnf partenariat, consulté dans sa version Kindle.

Queneau, Raymond, [1971] 1995, « De quelques langages animaux imaginaires et notamment du langage chien dans Sylvie et Bruno ", [L'Herne, Queneau, 1971], repris dans En passant, Éditions de L'Herne, coll. « Confidences », p. 99-111.

Restif de la Bretonne, Nicolas, [1781] 1990, La Découverte australe par un Homme-volant ou le Dédale français, dans Voyages aux pays de nulle part, Paris, Laffont, coll. « Bouquins », p. 1087-1261.

Swift, Jonathan, 1976, « Voyage au pays de Houyhnhnms », trad. fr. Jacques Pons, Voyages de Gulliver, Paris, Gallimard, coll. « Folio », p. 275-371.

\section{NOTES}

1. Une synthèse sur les langages des aliens prééexiste à son équivalent pour les langues imaginaires animales. Voir Landragin (2018). Pour un répertoire des langues animales, voir Albani et Buonarroti (2001).

2. Sophie Milcent-Lawson, Stylistique de la zoofiction française moderne et contemporaine. Représentation d'un point de vue animal dans la fiction française des $\mathrm{XX}^{e}$ et $\mathrm{XXI}^{e}$ siècles, à paraître. Ce travail s'inscrit dans le champ émergent de la zoopoétique, initiée au sein du programme "Animots» animé par Anne Simon. Voir le carnet de recherches https://animots.hypotheses.org.

3. Traduite en français par "suspension volontaire de l'incrédulité », cette expression empruntée à Coleridge est une notion utilisée en narratologie du discours fictionnel.

4. Pour pallier cette réticence - l'animal étant souvent défini comme un alogos, exclu du langage humain- la littérature invente d'autres dispositifs énonciatifs. Voir notamment Milcent-Lawson (2017). Anne Simon fait par ailleurs justement remarquer que: «De nombreux animaux ne sont pas de purs aloga et ne cessent de mettre en œuvre des stratégies de communication, d'en transmettre les modes d'une génération à l'autre ou, romanciers en puissance, de raconter des histoires en faisant prendre des vessies pour des lanternes à leurs congénères ou aux humains » (Simon 2017 : 72).

5. Nous empruntons ce néologisme à Jeandillou (1985:211).

6. Pour mémoire les deux volumes du Livre de la Jungle sont bien antérieurs (1894 et 1895), de même que les Histoires comme ça/Just so stories (1902), et aucune tentative de langue animale n'y était menée (si ce n'est dans l'épisode de Kotick, le phoque blanc, dont les paroles - «'Schoochnie! Ochen Scoochnie!' ('I'm lonesome, very lonesome!') said Kotick", The Jungle Book, p. 106 - sont en réalité une transcription phonétique du russe prononcé avec l'accent de îles aléoutiennes) de sorte que Paroles de chien manifeste un intérêt tardif chez Kipling pour les questions de transposition linguistique des langages animaux.

7. Traduction de Fanny Deleuze dans la Pléiade et dans la collection «Points romans » au Seuil (1992).

8. Elles sont plus nombreuses en anglais qu'en français : woof woof, arf arf, bow wow, bark bark, werf werf, ruff ruff.

9. En revanche, dans l'unique autre traduction disponible chez Bordas (dans un recueil de littérature de jeunesse intitulé La Revanche de Bruno, paru en 1982), Pierre Leyris a 
conservé le texte en doggee (traduit par canique), se contentant d'adapter la transcription graphique pour conserver la prononciation à l'anglaise : ainsi la première réplique devient : Oubouh, houh, bouhouyah! (p. 50). Les récurrences de morphèmes sont quant à elles strictement respectées.

10. Plus discutable m'apparaît la proposition selon laquelle « le fait le plus remarquable est l'existence d'un verbe "ne pas être" : Wooh, différent du verbe "être" Hah » (103).

11. Tout porte à croire que cette étude existe, on trouve trace de sa référence mais je n'ai pas réussi à la consulter.

12. Voir la série documentaire réalisée par Jérôme-Cécil Auffret, Paroles d'animaux, diffusée sur Arte les $1^{\text {er }}$ et 8 décembre 2018, disponible en ligne. Voir aussi Matignon (2018). Pour une synthèse documentée, voir Christen (2013: 141 et suiv.).

13. À l'exception notable du Dictionnaire des langues imaginaires [1994], de Paolo Albani, et Berlinghiero Buonarroti traduit aux Belles Lettres en 2001.

14. Voir Parmentier (2004).

\section{RÉSUMÉS}

Parmi les langues imaginaires inventées dans la fiction littéraire, les langues animales n'ont étonnamment pas, à ce jour, fait l'objet d'une investigation linguistique systématique. Pourtant, depuis l'Antiquité, avec Aristophane, jusqu'à la fiction ultra-contemporaine, en passant par les utopies des $\mathrm{XVII}^{\mathrm{e}}$ et $\mathrm{XVIII}^{\mathrm{e}}$ siècles, nombre d'auteurs ont fait le choix de ne pas se contenter de « donner la parole » aux animaux et ont tenté de forger des uglossies se démarquant des langues humaines. Les productions langagières prêtées aux diverses espèces (grenouilles, oiseaux, chevaux, lions, taureaux, ânes, phoques, poules, chiens, singes, etc.) se caractérisent dès lors par d'autres traits phonologiques, d'autres systèmes morphologiques, une autre grammaire.

Cet article se donne pour objectif de mener une réflexion sur les imaginaires langagiers en centrant le propos sur les représentations des langues animales imaginaires telles que la fiction littéraire a pu les concevoir au fil de diverses époques, de la satire à la fantaisie ludique en passant par la science-fiction. À partir de l'examen d'un échantillon d'énoncés "animaux " tirés de la littérature de fiction, on s'efforcera donc de dégager les caractéristiques de cette zoolinguistique imaginaire.

It is surprising that among the imaginary languages invented in fictional literature animal languages have not yet been subjected to a systematic linguistic investigation. And yet, from Aristophanes in antiquity through the Utopian literature of the seventeenth and eighteenth centuries to ultra-contemporary fiction, numerous authors have not been content to simply give a (human) voice to animals and have attempted instead to forge uglossias differing from human languages. These linguistic productions attributed to different species (frogs, birds, horses, lions, bulls, donkeys, seals, hens, dogs, monkeys...) are characterized by other phonological traits, other morphological systems, other grammars.

This article aims to conduct an inquiry into linguistic imaginaries by focusing on the representations of imaginary animal languages as they are evidenced in the fictional literature of different periods, from satire through science-fiction to ludic fantasy. Grounded in the analysis of a sample 
of "animal" utterances drawn from fictional literature, it will attempt to identify the characteristics of this imaginary zoolinguistics.

INDEX

Keywords : animal languages, imaginary languages, uglossia, fictional literature, anthropocentrism, logocentrism, phonocentrism, zoopoetics

Mots-clés : langues animales, langues imaginaires, uglossie, fiction littéraire, anthropocentrisme, logocentrisme, phonocentrisme, zoopoétique

\section{AUTEUR}

\section{SOPHIE MILCENT-LAWSON}

Université de Lorraine, EA 3476, Crem (Équipe Praxitexte) 